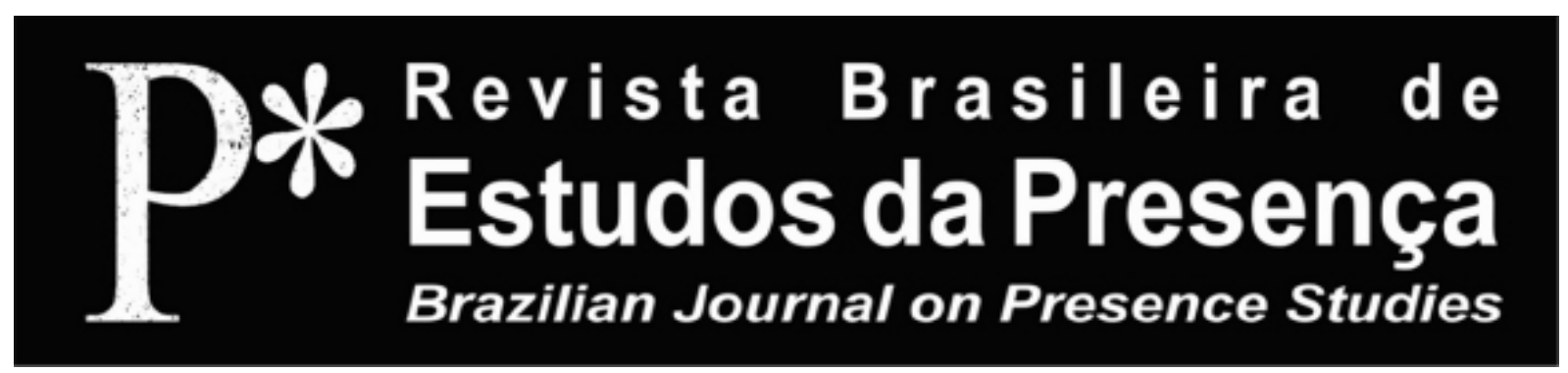

E-ISSN 2237-2660

\title{
Une Nouvelle Orientation Psychophysique dans la Pédagogie Théâtrale Contemporaine
}

\author{
Vezio Ruggieri \\ Università degli Studi di Roma La Sapienza - Rome, Italie
}

Lea Walter

Associazione Europea Psicofisiologi Clinici per l'Integrazione Sociale - Rome, Italie

RÉSUMÉ - Une Nouvelle Orientation Psychophysique dans la Pédagogie Théâtrale Contemporaine - Cet article a pour but de présenter comment les apports du modèle psychophysiologique bio-existentialiste élaboré par Vezio Ruggieri et ses collaborateurs peuvent jeter une lumière nouvelle sur les processus sous-jacents le jeu de l'acteur tels que la présence scénique, le processus d'identification avec un personnage et le complexe mécanisme de la prosodie. Cet encadrement théorique, qui voit le rapport corps-esprit dans une relation circulaire, éclairera le lecteur sur les bases physiologiques de la perception et de l'imagination ainsi que sur le rôle fondamental que la structure musculaire joue dans la construction de ces phénomènes.

Mots-clés: Psychophysiologie. Posture. Imagination. Émotion. Prosodie.

ABSTRACT - A New Psychophysical Approach in Contemporary Theater Pedagogy - This article aims at presenting how the contributions of the bio-existentialist psychophysiological model conceptualized by Vezio Ruggieri and his collaborators can bring a new light on some core elements underlying acting such as stage presence, identification process with a character and the complex mechanism of prosody. This theoretical mark, which sees the connection body-mind in a circular relation, will enlighten the reader on the physiological basis of perception and imagination as well as on the fundamental role that the muscular structure plays within the construction of these phenomena.

Keywords: Psychophysiology. Posture. Imagination. Emotion. Prosody.

RESUMO - Uma Nova Abordagem Psicofísica na Pedagogia Teatral Contemporânea - Este artigo tem como objetivo apresentar a forma como as contribuiçóes do modelo psicofisiológico bioexistencialista desenvolvido por Vezio Ruggieri e seus colaboradores podem trazer uma nova compreensáo sobre os processos subjacentes do jogo do ator, como a presença cênica, o processo de identificaçáo com um personagem e o mecanismo complexo da prosódia. Esse contexto teórico, que considera a conexáo corpo-mente como uma relação circular, esclarece ao leitor as bases fisiológicas da percepção e da imaginação, bem como o papel fundamental que a estrutura muscular desempenha na construção desses fenômenos. Palavras-chave: Psicofisiologia. Postura. Imaginação. Emoçáo. Prosódia. 


\section{Introduction}

Quels apports peuvent offrir les acquis de la recherche en psychophysiologieà l'étude et au travail sur la présence scénique de l'acteur et plus particulièrement sur le rapport entre l'identité de l'acteur et l'identité du personnage?Quelle est l'interaction entre l'organisation posturale, lefeeling et l'imagination dans le processus d'identification avec un personnage théâtral à interpréter? Comment favoriser le dialogue entre les plans imaginatifs, cognitifs, émotionnels, et les attitudes corporelles afin de garantir une réelle et visible liberté et qualité expressive de l'acteur? Enfin, de quelle façon la gestion des tensions musculaires organisant la posture est impliquée dans l'émission vocale et en particulier dans son aspect musical correspondant à la prosodie?

L'article cherchera àdonner des éléments de réponse à ces questions selon la perspective du modèle psychophysiologique bioexistentialiste conceptualisé par Vezio Ruggieri, au début des années 1980 auprès de l'Università degli Studi della Sapienza de Rome, qui a approfondi ces thèmes dans les volumes Identità in Psicologia e Teatro (2001) et Struttura dell'To tra soggettività e fisiologia corporea (2011). Ce modèle considère le Moi comme une unité psychophysique qui coordonne et module sa dimension corporelle (avec ses fonctions physiologiques élémentaires) ainsi que ses fonctions plus complexes, proprement dites psychologiques, telles que l'imagination, la perception, l'émotion et l'action. Selon cette vision, l'opération principale du Moi est de prendre conscience de soi, de son être au monde. Cette opération est le résultat d'un concret processus physiologiquequi procède à une synthèse unificatrice des informations provenant du monde extérieur et du monde intérieur (input) ainsi que des informations liées à la programmation et la réalisation de l'action (output). Il faut compter parmi les inputs l'ensemble des informations recueillies par le système sensoriel (vue, ouïe etc.), les informations concernant l'état des organes internes (sensibilité intéroceptive) ainsi que celles relatives au système musculaire qui revêt le corps (sensibilité proprioceptive). Dans cette perspective le Moipeut êtreconsidéré telleune sculpture vivante qui sert de pont entre l'input et l'output. Sans oublier que l'output (l'action), grâce à des mécanismes de feedback, agit, à son tour, en tant que input. Ce dernier point éclaire le lecteur sur la vision circulaire du rapport corps-esprit à la base du modèle psychophysiologique. Àce sujet, il est intéressant de relever que dans le 
langage quotidien le Moi, entendu comme objet de réflexion sur soi, est souvent représenté verbalement par la succession des deux termes $m o i, j e$. Cette particularité de l'usage français est curieusement très proche à la base de notre training de l'acteur qui commence par la prise de conscience du Moi comme processus d'auto-perception de soi qui résulte dans le $J e$ de l'acteur qui est le générateur de l'action. Nous appellerons donc Moi-Je cette instance self-conscious qui est à la fois génératrice et produit de l'interaction inexplicable des rapports corps-esprit (Ruggieri, 1988).

\section{La Posture comme Mise en Scène de l'Autoreprésentation de Soi}

La coordination des différentes dimensions que le Moi opère dans le contexte du jeu de l'acteur concerne le rapport entre la présence au monde et la présence scénique. Plus précisément, il s'agit de faire interagir demanière cohérente les attitudes posturales liées à l'organisation du tonus musculaire, avec l'autoreprésentation de soi qui se développe au niveau cérébral. On découvre ainsi que le tonus musculaire, outre sesfonctions de soutien et d'équilibre, joue un rôle morphogénétique fondamental en stabilisant de façon tonique certains rapports spatiaux entre les parties du corps qui définissent l'individualité expressive du sujet. La distribution des tensions musculaires est la base structurelle sur laquelle se nouent les composantes de la personnalité de l'acteur et les attitudes posturales liées aux rôles des personnages interprétés. Le système tonique musculaire, avant même d'être générateur de mouvement à travers l'activité motrice, est le point de raccord entre l'imagination, l'action et le sentir entendu à la fois comme feeling de base que comme forme spécifique d'émotions particulières vécues par le sujet.

La nouveauté conceptuelle réside dans le fait que les processus imaginatifs ainsi qu'émotionnels prennent forme au niveau cérébral mais conservent toujours une connexion avec la périphérie du corps. Pour autant, le modèle propose une pédagogie individualisée de l'acteur qui se concentre sur l'interaction circulaire des dimensions corporelles et imaginatives. Dans ce cadre, le langage verbal,conçu comme geste sonore, lie l'imaginaire au processus physiologique d'évènements acoustiques à travers un jeu de tension et solution de tension créant un clair-obscur essentiel pour la compréhension du message linguistique (Ruggieri, 2010). Ce processus se situe lui aussi dans le contexte de la structure du Moi-Je et de ses identités (identités de 
base et sous-identités correspondantes aux rôles). L'identité du Moi-Je se construit à partir de l'interaction entre l'autoreprésentation cérébrale du Moi et la posture de base. En d'autres mots, la posture est la mise en scène corporo-spatiale de l'autoreprésentation de soi qui se forme au niveau cérébral. La posture est donc à la base de notre relation au monde.

Cette conception, qui revisite certaines théories psychologiques longtemps obsolètes mais ravivées de nos jours (Lowen, 1978), exige un approfondissement sur le thème spécifique de la construction de l'identité selon le modèle bio-existentialiste. L'identité est issue de l'interaction dynamique entre l'identité nucléaire de base et les sousidentités. Àtitre d'exemple, dans la vie quotidienne, un sujet lie à son identité de base ses différentes sous-identités qui peuvent émerger dans des contextes ou situations particulières telles que la sous-identité de fils, mari, employé etc. Chacune de ses sous-identités est non seulement liée à un processus d'autoreprésentation mentale mais présente des spécificités dans les attitudes posturales et interpersonnelles. Au théâtre, le processus d'identification avec un personnage implique l'assomption d'une nouvelle sous-identité suggérée par l'imagination et/ou le texte. L'identité est liée à un double miroitement entre cerveau et périphérie du corps. Le corps construit, à travers les voies afférentes, une représentation de l'image corporelle qui est à son tour le point de départ de l'activité tonique qui donne forme à la posture, à travers les voies efférentes. Ce mécanisme à double miroitement dans lequel une posture est la mise en scène de l'autoreprésentation, fonctionne pour la construction de l'identité nucléaire ainsi que pour les sous-identités liées à un personnage théâtral. Sans oublier que pour chacune de ces autoreprésentations de soi correspond un feeling généré par le jeu de tensions musculaires.

\section{Comment se Construit le Sentiment de Présence?}

Le thème de la présence scénique est fondamental dans le travail de l'acteur. Pour la majeure partie des personnes, cette dimension a quelque chose d'imprévisiblement magique, difficilement analysable et encore plus difficilement imaginable comme un élément sur lequel on peut travailler consciemment à travers une pédagogie de l'acteur. L'analysabilité du phénomène apparait en adoptant un nouveau point de départ qui conçoit différemment les rapports entre le corps et l'esprit. Lapproche psychophysiologique conçoit en effet le Moi- 
Je comme unité psychophysique dans laquelle les composantes dites corporelles et celles dites psychologiques (imagination, processus cognitifs, émotions etc.) se relient directement avec l'activité physiologique du corps et avec ses fonctions que l'on appelle biologiques élémentaires.Selon cette vision, le Moi-Je est un système fonctionnel paradoxal car s'il naît et prend forme à travers l'interaction des fonctions physiques et psychiques, il est en même temps le point de raccord ainsi quacteur de la régulation, modulation et stimulation deces mêmes fonctions.

Cette conception moderne revisite de manière profonde certains aspects de la physiologie traditionnelle en donnant pour acquises les analyses universellement partagées concernant le fonctionnement des différents organes et systèmes. La nouveauté de cette vision du Moi-Je réside dans l'introduction du concept d'intégration fonctionnelle qui est à la base des fonctions psychologiques complexes par le biais d'un processus d'interaction des sous-unités fonctionnelles physiologiques. C'est une physiologie qui se concentre sur les connexions entre les systèmes et sur les connexions entre les connexions. La littérature scientifique contemporaine montre un intérêt toujours plus croissant au rôle du cerveau dans cette dynamique d'intégration d'interconnexions (Fery, 2001/2002). Dans cette optique, certains auteurs (Damasio, 2010) identifient explicitement le Moi avec le cerveau en créant ainsi une confusion entre fonction et structure. $S$ 'il est vrai que le cerveau est constitué de parties spécialisées dans le développement de fonctions psychologiques, comme par exemple l'aire du cortex visuel qui joue un rôle déterminant dans la vision d'images et par conséquent dans le processus de l'imagination (Argyle; Dean, 1965). Nous pouvons affirmer que les images deviennent images visibles seulement si elles sont vues par une structure plus complexe, telle que le Moi-Je qui est un système fonctionnel de raccord de toutes les fonctions cérébrales ainsi que de la relation corps-esprit.

Àpartir de ce point, on rentre directement dans le thème de la présence scénique, auquel on s'approche en considérant deux points nodaux: le premier lié à la relecture moderne des processus perceptifs et le deuxième concernant les phénomènes des représentations mentales et des vécus émotionnels. Cette relecture fondamentale introduit le concept de la conscience d'une manière générale et de la propre conscience d'être au monde ( $D a$ Sein) comme élément porteur de la présence scénique en termes purement perceptifs. 
Sitraditionnellement, la recherche expérimentale a examiné les processus de perception des différents apparats sensoriels, selon le modèle psychophysiologique, la conscience n'est autre que le vécu d'intégration unifiée des informations provenant des différents systèmes sensoriels en fonction du Moi-Je. Autrement dit, le Moi-Je naît de l'intégration des perceptions élémentaires qui s'intègre les unes avec les autres. Le Moi-Je n'est donc pas uniquementla somme de la perception issue de la vue, de l'ouie, du toucher, du goût et de la proprioception (sensibilité des muscles), mais il est le résultat de l'unification de ses sous-fonctions. Le rapport aux expériences sensorielles, cette perception intégrale que nous appelons conscience, représente la base des différents actes perceptifs que le Moi-Je peut moduler.

\section{Perception et Imagination se Rencontrent dans la Représentation}

Un discours analogue est appliqué à l'étude de l'espace (Ruggieri; Cocchia, 2012). Là aussi, l'approche purement physiologique entend l'espace comme une réalité autonome et indépendante du Moi-Je. Le modèle psychophysiologique, sans entrer en contradiction avec le sens commun, étudie les modalitésà travers lesquelles l'être humain génère et construit activement sur le plan perceptif l'expérience de l'espace. Selon cette perspective, le Moi-Jetravaille en deux temps, ou plutôt à deux échelles: il construit l'espace dans lequel les évènements sont perçus de même qu'il construit son propre espace corporel (Ruggieri; Thellung, 2000). Cet espace-corps qui est une structure unitaire, unifié et individué par rapport à l'environnement est le fond de base dans lequel s'insèrent les actes perceptifs et représente aussi la structure à partir de laquellese déploie l'expressivité (Ruggieri, 1997).

L'expressivité, selon ce point de vue, est composée de figures motrices et manipulatoires propres à la relation sujet-environnement. Le point important sur lequel ouvrir une réflexion concerne ce processus d'unification de l'expérience subjective de l'espace lié à l'expressivité. Ce qui amène à se pencher sur la conception du MoiJe comme pont entre le système perceptif et le système moteur-expressif (dont le langage verbal est une des fonctions les plus importantes). Concrètement, ce point de départ amènera à démontrer comment ces deux systèmes sont étroitement liés en partant de l'explication du passage fonctionnel qui va du physique au psychique. 
Ce passage crucial s'opère déjà dans l'acte perceptif qui consiste à traduire une stimulation externe en une représentation interne qui reproduit la forme de l'évènement perçu par le biais des capteurs sensoriels. Les études sur les procédés cognitifs (Ruggieri, 2011; Wilson, 2002), dont la recherche théâtrale fait de plus en plus référence, conçoivent comme une évidence cette opération et se concentrent sur l'élaboration cérébrale du matériel déjà traduit - étude du développement du langage, processus d'abstraction, symbolisation etc. (Damasio, 2010). Le premier phénomène prodigieux réside dans le fait que le monde externe est représenté, réécrit, pour faire partie du monde interne. C'est bien sur cette réécriture que les ultérieures élaborations adviennent. Il est stupéfiant de penser que des énergies électromagnétiques se transforment en représentations internes formant des images. Ceci amène à poursuivre la réflexion, qui a été documentée au niveau expérimental (Ruggieri, 1997), en affirmant que «[...] les représentations dérivées de l'acte perceptif sont physiologiquement identiques aux représentations qui peuplent notre univers imaginatif "(Ruggieri, 1999, n.p.). Ce nouveau point de vue prend ses racines à partir des recherches de l'école de Kosslyn (1994) qui a démontré en premier que les aires du cerveau impliquées dans la production d'évènements visuels jouent un rôle analogue dans la production d'évènements imaginatifs. L'imagination, qui suit un mécanisme physiologique semblable à celui de la perception, acquiert un nouveau sens fonctionnel car elle devient instance organisatrice du comportement. Dans ce cas, on parle d'imagination programmatrice. Selon cette vision, l'imagination n'est plus en opposition au phénomène perceptif. Elle joue un rôle fondamental dans l'organisation et la réalisation de l'action. En effet, chaque acte présuppose une activité cérébrale qui, non seulement prend en compte les informations perceptives (représentation mentale du contexte physique dans lequel se déroulera l'action), mais anticipe visuellement l'action que l'on entend réaliser. Si l'on considère que l'autoreprésentation du Moi est une forme particulière d'imagination (représentation mentale produite par le cerveau), on peut affirmer qu'elle est à la base de l'organisation posturale de base et de ses attitudes.

Ramener sur le même plan physiologique les processus perceptifs et imaginatifs ouvre des possibilités d'application dans l'art théâtral en travaillant sur les connexions entre la construction des expériences liées à la relation avec le monde extérieur, et la formation 
des représentations internes auto-générées par le texte par exemple. Ces dernières peuvent surgir - en fonction du Moi-Je -soit du dépôt biochimique de la mémoire neuronale soit d'une nouvelle production d'images, jamais vues auparavant, propres au processus de la créativité. Ce que la psychanalyse traditionnelle appelle inconscient entre parfaitement dans ce cadre, s'agissant d'un ensemble de représentations déposées dans la mémoire mais qui sont difficiles d'accès par le Moi-Je qui voudrait les faireréapparaitre sur le plan de la conscience. Pour expliquer concrètement cette connexion entreces différents plans, on peut prendre à titre d'exemple le travail de mise en scène où l'imaginaire du texte théâtral rencontre la représentation de ce qu'il adviendra sur scène (spectacle comme façon de donner à voir l'imaginaire). L'art réside dans l'intégration de ces deux niveaux de processus en une vérité qui s'exprime sur un plan concret, spatial et phénoménologique.

\section{L'Activité Musculaire au sein du Rapport entre Imagination et Sentiment}

Avant même d'affronter le thème de la présence scénique nous devons considérer un autre aspect de l'expérience perceptive. Quand le läqque approche lethème de la perception,celle-ci estsouvent réduite aux cinq sens, sans considérer la sensibilité de l'activité musculaire qui est fondamentale. Le muscle est un producteur de mouvements et d'activité tonique indispensables pour l'équilibre postural. La proprioception (sensibilité des muscles)est aussi source d'informations pour le système nerveux central (SNC) et pour le plus grand système fonctionnel appelé Moi-Je, sur l'exécution de son activité motrice ainsi que sur son activité tonique.En effet, outre la contraction isotonique qui détermine le raccourcissement du muscle et qui produit le mouvement, il existe aussi une contraction isométrique dans laquelle le muscle, fixé dans ses deux extrémités, produit une augmentation de tension mais pas de mouvement. Cedernier type de tension est perçu par le sujet et devient la base d'un vécu de présence, dans lequel le corps mêmeinforme le Moi-Je de son niveau de tension interne. Lactivité musculaire opère donc un autre passage fonctionnel intéressant car elle indique, à travers la narration de ses tensions, l'arrière-plan émotionnel du Moi-Je. La nouvelle stupeur naît exactement du fait que les senseurs mécaniques des muscles nous informent sur notre état de plaisir, douleur et tension interne sans générer pour autant des 
représentations mais en faisant référence à un vécu sentimental qui se situe dans l'axe plaisir-douleur. La proprioception nous renseigne sur les conditions d'intégrité ou de partielle désarticulation de cette unité que nous appelons corps. Puisque le jeu des tensions musculaires présente une gamme infinie de variations possibles, on comprendra que l'adoption de ce point de vue permet une compréhension différente des vécus qui constituent l'arrière-plan sentimental du Moi-Je. Prenons par exemple les sensations de légèreté, de lourdeur ou de rigidité, ne sont-elles pas une perception subjective de la modalité d'organisation des tensions musculaires que le Moi-Je opère et dont il est paradoxalement le premier témoin, générant ainsi sa manière spécifique d'y être (Da Sein)? En revisitant la conception de Lange (1887) et James(1890), on peut affirmer que l'expérience perceptive liée à l'activité musculaire est génératrice du sentir subjectif, à savoir le feeling. La dynamique sensorielle, contribuant à la construction du feeling,se différencie formellement du processus de perception universellement reconnu que l'on appelle analogique (Ruggieri, 2011). En ce qui concerne le phénomène perceptif, la représentation mentale produite suite à la traduction du stimulus visuel ou acoustique reflète, au niveau du cortex, la forme de l'objet-stimulus. Parallèlement à ce processus analogique de la perception, il s'opère un processus qui confère une connotation émotionnelleau stimulus en question générée par le jeu des tensions tonique-musculaires. Pour autant, la dimension imaginative possède des connexions étroites avec l'apparat sensoriel-moteur du corps. Cette connexion est donc le noyau porteur de la mise en scène dans laquelle se lient de manière cohérente les dimensions dénotatives (processus analogique) et connotatives (processus émotionnel). C'est bien dans cette fusion de niveaux que se construit l'unité et l'authenticité expérientielle sur laquelle s'attarde Stanislavski. Le jeu de l'acteur paraîtra mécanique et perdra de sa force expressive quand le plan corporel de l'action ne se lie pas à l'imagination qui est à la base de sa production ou quand l'imagination même perdra ses connexions avec l'organisation sensoriel-moteur à la base du feeling lui-même lié aux tensions musculaires.

Maintenant que le phénomène perceptif est encadré et que l'on a souligné comment l'activité motrice qui produit les mouvements et les gestes envoie au Moi-Je des messages sur sa propre activité, on peut à nouveau aborder le thème de la présence scénique entendue comme perception intégrée de soi liée à l'autoreprésentation du 
Moi-Je. Cette autoreprésentation est un chapitre de l'imagination construite sur la base d'une auto-perception générée par la posture. Àson tour, l'autoreprésentation a une fonction génératrice qui détermine le niveau d'activité tonique des différentes parties du corps responsable de la posture même. Cette dynamique éclaire de façon précise la circularité des rapports corps-esprit.

Pour synthétiser ce discours, on peut se référer au postulat cartésien "Cogito ergo sum ${ }^{1}$ » (Descartes, 1840, n.p.). Le modèle psychophysiologique retourne cette affirmation car il démontre que c'est bien parce que je suis (présent à moi-même) que je suis conscient et capable de mettre en marche des fonctions de l'être telles que penser, marcher, aimer etc. Le sentiment de base d'exister, d'y être, ne peut donc pas être précédé par les fonctions mêmes de l'être.

En revenant sur le sentiment de présence, abordons le processus qui construit cette conscience d'y être, à savoir, d'être dans le présent.Le processus psychophysiologique à la base de la représentation d'être dans le présent est aussi une opération de coordination mise en œuvre par le Moi-Je. La sensation de simultanéité est rendue possible grâce aux circuits réverbérants de la mémoire qui, de par leur forme circulaire, permettent aux informations perceptives (internes et externes) de rester en circuit pendant un certain temps.De cette façon, se construit l'illusion d'être dans le présent, alors que concrètement, ce sont des informations passées qui se somment. Pour simplifier, nous pourrions dire que je me sens dans le présent car j'ai mémoire de moi. Ces circuits de la mémoire neuronale sont les mêmes utilisés pour la construction de la représentation de l'espace investi. Il est évident que l'ensemble de ces processus estau-dessous de la conscience, néanmoins c'est bien à partir de ces vécus de présence que l'on peut agir dans le monde.

\section{L'Inhibition et le Contrôle de l'Émotion dans l'Expressivitéde l'Acteur}

Une dimension fondamentale dans l'éducation somatique de l'acteur est l'expression des émotions. Quel est le processus psychophysiologique sous-jacent auxémotions et à leur expression? Une réponse émotionnelle suit la séquence suivante: un stimulusémotigène(interne ou externe), susceptible de provoquer une émotion, stimule des parties spécialisées du cerveau (hypothalamus, système limbique) qui à 
leur tour produisent des spécifiques patterns comportementaux. La plupart des émotions sont des réponses à la base de processus plus amples liés aux comportements instinctifs. La colèrepar exemple: celle-ci représente la phase préparatoire du comportement agressif (Ruggieri,1988) et joue la fonction d'auto-signal pour le sujet qui module la réponse agressive. Le sentiment subjectif de la colère est produit par le jeu de tensions musculaires généré par le système nerveux central. Ce type de description de la dynamique émotionnelle est particulièrement intéressant pour comprendre le mécanisme d'inhibition et de contrôle qui, souvent, empêche l'acteur d'exprimer librement ses intentions expressives. Puisque le sentiment est l'expression de l'activité musculaire, selon l'approche psychophysiologique, l'inhibition correspond à une excessive augmentation de tension musculaire qui peut devenir un blocage: une contracture. La dynamique musculaire perd ainsi la capacité de produire des signaux et raidit certaines parties corporelles de variable extension afin de bloquer l'émotion. Les muscles à travers leur activité tonique sont tous reliés les uns aux autres, que cela soit dans une même zone ou dans des zones contiguës, où ils adoptent une fonction de raccord entre les articulations (jonctures). Malgré le fait qu'il puisse y avoir des différences de tension entre les différentes parties du corps, les interconnexions constituent un réseau ou une toile qui enveloppe tout le corps en produisant une sensation d'unité, base du sentiment de présence. Cela-dit, il est possible que la connexion entre les différentes parties du corps soit interrompue par des contractures, qui ne sont rien d'autre qu'une tension non suivie de relâchement. De cette façon certaines parties du corps seraient exclues de la formation d'un cadre unitaire ainsi que de la participation active au feeling de base allant influencer négativement la présence scénique liée à l'auto perception intégrée des tensions musculaires. Pour autant il est nécessaire dans un premier temps de procéder à une analyse des distributions des tensions posturales et de la cohérence des différentes parties du corps à partir desquelles naissent les actes singuliers. Dans cette phase, on identifie les contractures et on enseigne au sujet-acteur à rétablir l'équilibre de base en adoptant une nouvelle gestion du poids qui demande une réorganisation de l'ensemble de la posture. En effet, ces contractures ou interruptions empêchent le passage fluide des tensions musculaires d'une partie du corps à une autre en rendant plus complexe le passage du poids dans la dynamique de l'auto-appui. 
Dans l'analyse et le travail sur la posture et les contractures musculaires qui la composent, nous pouvons identifier ces mécanismes d'inhibition des émotions que le sujet, l'acteur, porte en luila plupart du temps depuis toute sa vie. Ce dernier concept est de fondamentale importance pour le travail d'éducation somatique de l'acteur qui vise à libérer le sujet des tensions interférentes à l'expressivité. Plus l'acteur est libre des tensions inhibitoires, plus sa palette expressive sera riche. Néanmoins, cette opération de solution des tensions, non seulement ne peut être conduite de façon mécanique, mais peut être extrêmement dangereuse pour la stabilité de la structure du Moi-Je. En effet, ces inhibitions sont des mécanismes de défense que le sujet a mis en place pour maintenir son intégrité psychophysique. Cela représente en somme sa manière spécifique d'être au monde. Il est donc essentiel que la pédagogie de l'acteur prenne en compte ces dimensions existentielles en l'encadrant dans un processus qui agit à la réorganisation constructive du Moi-Je à partir de l'organisation posturale.

\section{Le Training de l'Acteur comme Construction d'une Identité Psychophysique entre Réalité et Imagination}

Comme dit auparavant, la posture, miroir reflétant l'autoreprésentation de soi, se situe dans les grandes catégories de stabilité et de flexibilité qui doivent s'harmoniser afin de garantir une réelle liberté et une qualité expressive. Àce sujet, il est extrêmement intéressant que le thème de stabilité et flexibilité, caractéristiques de l'identité, peut être traité sur le versant psychocorporel. Le premier principe du training de l'acteur selon cette vision est de construire des expériences pédagogiques autour de la dynamique physiologique de l'appui qui est à la base du maintien de l'équilibre postural. Concrètement, il s'agit de proposer une série d'expériences intermédiaires (d'hétéro-appui) qui amène l'acteur à se sentir suffisamment confiant afin de pouvoir lâcher son poids au sol sans le retenir dans telle ou telle autre partie du corps (auto-appui). Dans ce cadre, le thème psychophysique de l'appui acquiert le rôle de métaphore concrète autour du thème de stabilité et flexibilité de la structure du Moi-Je. L'auto-appui devient une catégorie psychophysique car elle rentre dans la dimension de la confiance que l'acteur possède en lui-même. Cette confiance s'exprime à travers une organisation et une gestion des tensions musculaires dans lesquellesla force du poids joue un rôle central.Une des 
expériences intermédiaires consiste à demander au sujet s'il observe des différences en termes de feelings et d'organisation posturale quand il prononce intérieurement J'ai un corps ou Je suis mon corps. Cette expérience peut être le début d'un travail plus approfondi sur les autoreprésentations possibles ainsi que sur l'apparat tonique-moteur qui prend forme dans la posture. Ce type d'exercices vise à lier les différentes dimensions imaginatives avec la capacité dynamique de moduler la décharge du poids vers un auto-appui. Àla suite d'un travail pédagogique répété, l'appui expérimenté physiologiquement peut trouver son correspondant dans la représentation corticale et acquérir une forme constante dans le temps, en allant modifier ainsi la structure psychophysique de l'acteur.

Le théâtre conduit à faire interagir les dimensions imaginative, perceptive et motrice tout en soulignant le fait que ce sont des aires opérationnelles du Moi-Je toujours physiologiquement en interaction. Le travail pédagogique de l'acteur peut faire en sorte que ces dimensions se coordonnent harmoniquement entre elles, en formant une plateforme pour une libre créativité expressive. Le travail se concentre principalement sur le rapport entre l'identité nucléaire de base de l'acteur et l'identité du personnage à interpréter. Le jeu apparaîtra artificiel ou mécanique quand manquera une cohérence entre représentation mentale et phénoménologie corporelle. Souvent, ce manque de cohérence est dû à une posture habituelle de l'acteur qui ne laisse pas assez d'espace à la posture liée au personnage: la représentation mentale ne réussit pas à investir le corps dans son ensemble. Le but du training est celui d'offrir des clés à l'acteurpour que son tissu musculaire puisse travailler harmoniquement dans la production de postures fonctionnellement unitaires en rapport aux différentes représentations mentales. Selon cette vision, l'expérience théâtrale consiste à se placer dans des réalités imaginaires complètement différentes ou plutôt de donner une réalité physique à une réalité imaginaire. On considère un bon jeu d'acteur lorsqu'il est capable de changer les coordonnées spatiales et représentationnelles-imaginatives dans lesquelles il développe son action substantiellement différente du quotidien, tout en s'appuyant sur les concrets piliers spatio-temporels investis par la nouvelle forme de l'imaginaire. L'acteur en action se trouve à vivre deux possibles univers: celui de sa réalité personnelle, issu de son identité nucléaire (de sa propre et unique façon d'être au monde) et celui de l'univers imaginaire et perceptif lié à la sous-identité du 
personnage à interpréter (avec ses attitudes posturales différentes). Le training de l'acteur vise à développer cette particulière capacité à passer d'une dimension à une autre. Celle-ci est étroitement liée à la gestion et la modulation des tensions musculaires qui n'est pas une question mécanique car les muscles sont eux-mêmes générateurs de la production imaginative et du feeling.

\section{La Prosodie, La Danse des Mots}

Le thème de la physiologie de l'appui, qui est relié directement à ceux du rythme et des mécanismes plus sophistiqués de la régulation des tensions musculaires, nous donne l'opportunité d'aborder la multiplicité des rythmes qui caractérisent les univers corporels. Il est particulièrement intéressant d'observer la relation entre la posture de base et la dynamique rythmique de la prosodie propre du langage verbal. Àce propos, nous considérons la parole et la phrase comme des gestes sonores qui fleurissent dans le contexte d'une posture et de sa régulation rythmique de base. La régulation des différents rythmes s'opère à travers une modulation des tensions posturales toniques (Ruggieri, 2011). S’il est facile de comprendre l'automaticité du passage de la musique à la danse dans toutes les cultures, nous pouvons penser, à partir d'un sentir semblable, que dans les mots et dans le rythme des phrases se cache une danse. Autrement dit, en inversant l'ordre de cette pensée, nous pouvons imaginer une danse capable de générer le rythme d'une phrase. Il est aussi important de souligner à ce sujet que le rythme prosodique, avec son jeu d'accent et de tensions, exerce bien plus qu'une pure fonction esthétique etcontribue à donner sens et spécificité aux dimensions logique, cognitive et grammaticale propres d'une phrase (Chomsky, 1965). Ici, l'objectif du training, selon l'approche psychophysiologique, est celui de danser le texte, c'est-à-dire de faire coïncider les accents d'une phrase avec la chute du poids et sa réponse de retour automatique que l'on peut assimiler à un rebond. Ceci implique un travail de modulation des tensions musculaires qui permet cette réponse élastique. Ici aussi le Moi-Je doit coordonner harmoniquement le plan imaginatif lié au texte et le plan tonique-musculaire lié à la gestion du poids en correspondance aux accents suggérés par le texte. 


\section{Conclusions}

Cet article a présenté une pédagogie de l'acteur qui est strictement liée à un récent cadre théorique étudiant la structure psychophysique du Moi conçu comme coordinateur d'une part, de l'activité corporelle, et d'autre part de la subjectivité. Il est à la base de toutes les principales actions de l'acteur dans lesquelles se nouent l'imagination, le feeling, le mouvement et la présence scénique. La conscience des interactions physiologiques entre les fonctions du Moi (Moi-Je) permet d'analyser, dans le cadre de l'étude des attitudes posturales habituelles, les différences individuelles, en explorant les inhibitions et les potentialités expressives de chacun. En partant de cette analyse, l'objectif du training de l'acteur sera de produire des modifications de la structure psychophysique en fonction des intentions esthétiques de la mise en scène. Se rendre disponible, selon cette perspective, est un processus concret qui concerne la disponibilité corporelle et imaginative à représenter phénoménologiquement, à travers la modulation des tensions musculaires, les personnages que l'on veut interpréter. L'éducation somatique de l'acteur passe par une réorganisation posturo-spatiale qui permet un investissement de toutes les parties du corps en cohérence avec l'imaginaire et le texte.

Cet article a voulu aussi démontrer comment le modèle psychophysiologique bio-existentialiste est structurellement interdisciplinaire. En effet, l'encadrement qu'il propose peut facilement dialoguer avec les traditions pédagogiques de l'acteur les plus importantes et les plus reconnues - comme la tradition grotowskienne qui a mis en valeur la dimension psychocorporelle de l'acteur - ainsi qu'avec les pratiques théâtrales qui privilégient la parole ou la gestualité abstraite qui ne peuventse soustraire à la confrontation avec l'organisation psychophysique du Moi-Je. Ce dialogue est possible car ce modèle situe dans un unique cadre physiologique les différentes fonctions du Moi-Je qui coordonne circulairement ses propres fonctions physiques et psychiques. Notre conception rejoint celle de Pradier (1997) qui élargit énormément l'espace opérationnel du théâtre et nous pousse encore une fois à souligner l'analogie des mécanismes psychophysiques propres des acteurs lors d'une mise en scène avecceux de l'homme qui met en scène sa vie personnelle dans le quotidien.

Vezio Ruggieri; Lea Walter - Une Nouvelle Orientation 


\section{Notes}

1 "Je pense donc je suis" (Descartes, 1840, n.p.).

\section{Références}

ARGYLE, Michael. Il Corpo e il suo Linguaggio. Bologna: Zanichelli, 1978.

ARGYLE, Michael; DEAN, Janet. Eye-Contact, Distance and Affiliation. Sociometry, Washington, D.C., American Sociological Association, v. 18, n. 23, p. 289-304, set. 1965.

BARBA, Eugenio. La Canoa di Carta. Bologna: il Mulino, 1993.

CHOMSKY, Noam. Aspects of the Theory of Syntax. Cambridge: MIT Press, 1965.

DAMASIO, Antonio. Self Comes to Mind:constructing the conscious brain. New York: Pantheon, 2010.

DESCARTES, René. Discours de la Méthode. Paris: Hachette, 1840.

FERY, Yves-André. Que Savons-nous de nos Mouvements? STAPS, Paris, Université René Descartes-Paris V, n. 55, p. 7-22, 2001/2002.

FISHER, Seymour. Development and Structure of the Body Image. London: Lawrence Erlbaum Associates Publishers, 1986.

JAMES, William. The Principles of Psychology. New York: Holt, Rinehart and Winston, 1890.

KOSSLYN, Stephen Michael. Image and Brain:the resolution of imagery debate. Cambridge: MIT Press, 1994.

LANGE, Carl Georg. Uber Gemuts-Bewegungen. Leipzig: Kurell, 1887.

LOWEN, Alexander. Il Linguaggio del Corpo. Milan: Feltrinelli, 1978.

MORRIS, Desmond. L'Uomo e i suoi Gesti. Milan: Mondadori, 1978.

PRADIER, Jean-Marie. La Scène et la Fabrique des Corps:ethnoscénologie du spectacle vivant en Occident. Bordeaux: Presses Universitaires de Bordeaux, 1997.

RUGGIERI, Vezio. Bodily Perception in the Organization of Postural Attitude and Movement. Perceptual and Motor Skills, Missoula, AmSci Publications, n. 82, p. 307-312, 1996.

RUGGIERI, Vezio. L'Esperienza Estetica:fondamenti psicofisiologici per un'educazione estetica. Rome: Armando, 1997.

RUGGIERI, Vezio. L'Identità in Psicologia e Teatro: analisi psicofisiologica della struttura dell'io. Rome: Edizioni Scientifiche Magi, 2001.

RUGGIERI, Vezio. Immaginazione e Percezione si Incontrano nello Sguardo. Realtà e Prospettiva in Psicofisiologia, Naples, Edizione Scientifiche, n. 5-6, p. 119-131, 1993.

RUGGIERI, Vezio. Mente, Corpo e Malattia. Rome: Il Pensiero Scientifico, 1988. 
RUGGIERI, Vezio. On the Hypothesized Correspondence between Perceptual and Imagery Process. Perceptual and Motor Skills, Missoula, AmSci Publications, n. 73, p. 827-830, 1991.

RUGGIERI, Vezio. Relationship between Spatial Experiences, Narcissistic Dimensional Development and Posture. Rome: International Conference on Spatial Cognition, 2000 .

RUGGIERI, Vezio. The Running Horse Stops: the hypothetical role of the eyes in imagery movement, Perceptual and Motor Skills, Missoula, AmSci Publications, n. 89, p. 1088-1092, 1999.

RUGGIERI, Vezio. Il Ruolo del Ritmo e della Semantica di una Poesia nella Produzione di Emozioni Estetiche. Attualità in Psicologia, Rome, Università di Roma,n. 1, p. 17-26, 1986.

RUGGIERI, Vezio. A Zoom Lens before the Eyes during Imagery: individual differences and strange unexpected responses. Perceptual and Motor Skills, Missoula, AmSci Publications, n. 78, p. 451-454, 1994.

RUGGIERI, Vezio; COCCHIA, Carla.Space Contraction Experiences during Acoustic Metronomic Stimulation: a synthetic discussion. Cognitive Processing, Berlin, v. 13, n. 1, supplement, p. 309-311, ago. 2012.

RUGGIERI, Vezio; DELla GIOVAMPAOLA, Sara. About Spatial visual imagery. Rome: International Conference on Spatial Cognition, 2000.

RUGGIERI, Vezio; THELLUNG, Marina. Personal space as real concrete phenomenon. Rome: International Conference on Spatial Cognition, 2000.

RUGGIERI, Vezio et al. Struttura dell'Io tra Soggettività e Fisiologia Corporea. Rome: Edizioni Universitarie Romane, 2011.

SHILDER, Paul. Immagine di sé e Schema Corporeo. Milan: Feltrinelli, 1980.

WILSON, Margaret. Six Views of Embodied Cognition. Psychonomic Bulletin \& Review, Berlin, Psychonomic Society, v. 9, n. 4, p. 625-636, 2002. 
Vezio Ruggieri est professeur de Fondements Psychologiques des Arts du Spectacle auprès de la faculté de Lettres et Philosophie de l'Université de Rome La Sapienza (UniRoma1). Il dirige un Master en Art-thérapie depuis 2002. Il a été professeur de Psychophysiologie Clinique auprès de la Faculté de Psychologie (UniRoma1) de 1972 jusqu'en 2013. Il a conduit de nombreux séminaires de théâtre selon son modèle et a dirigé un long métrage tiré de son roman sur la philosophe Ipazia ainsi qu'un court métrage sur Kafka.

E-mail: vezio.ruggieri@uniromal.it

Lea Walter est psychologue clinicienne spécialisée en art thérapie selon le modèle psychophysiologique clinique. Sa thèse de fin de Master auprès de la chaire de Vezio Ruggieri portait sur le lien entre l'archétype et l'expressivité corporel. Depuis 2007, elle collabore à des recherches d'ethnopsychiatrie en Afrique de l'Ouest où elle coordonne aussi des projets d'échange artistique. Elle pratique le théâtre en Argentine, Italie, France (avec Marc'O). En 2014 a conduit un projet de théâtre communautaire pour l'Espace Michel Simon.

E-mail: leawalter.psi@gmail.com

Ce texte inédit, révisé par Annelyse Gayraud, est également publié en portugais dans ce numéro. 